\title{
Extraordinary Directive Emission and Scanning from an Array of Radiation Sources with Hyperuniform Disorder
}

\author{
Orestis Christogeorgos $\odot,{ }^{*}$ Haoyang Zhang $\odot,{ }^{\dagger}$ Qiao Cheng, ${ }^{\dagger}$ and Yang Hao $\overbrace{}^{\S}$ \\ School of Electronic Engineering and Computer Science, Queen Mary University of London, London E1 4NS, \\ United Kingdom
}

(Received 29 March 2020; revised 26 November 2020; accepted 12 January 2021; published 29 January 2021)

\begin{abstract}
The main challenge in the antenna or laser array design is to find the element distribution that best meets the optimal performance for broadband emission and large angle beam steering. In the past, the design strategy was restricted to arrays with periodic, aperiodic, and random distributions, which are characterized by several fundamental limitations related to the operating frequency, the power consumption that arises from interelement interference, and the computation time required during the random optimization process. Furthermore, the interelement spacing has a lower or upper bound due to the elements' physical dimensions and the former prohibits the use of the aforementioned element distributions for small operating wavelengths, whereas the latter induces high-order grating lobes. We prove that hyperuniform disorder is an array element distribution evolving through natural selection processes that warrants a disordered solution to the array design when this is treated as a packing problem. We theoretically and experimentally report that the array with hyperuniform disorder exhibits extraordinary directive emission and scanning features, while being scalable for extra-large arrays without any additional computational effort.
\end{abstract}

DOI: 10.1103/PhysRevApplied.15.014062

\section{INTRODUCTION}

Directive emission with beam scanning has been the main motivation of phased array design, the concept of which has been extended from microwave to optics [1,2] for the coherent control of optical emission by shaping the phase of laser pulses. The mathematical principle of the phased array is based on wave diffraction physics, in which the radiation field results from the coherent addition of all radiation sources in the array, calculated by adding the desired phase shift to the fringing term. Although they do not explicitly require specific element distribution specifications, traditional phased arrays have been largely based on periodically ordered distributions due to their mathematical simplicity. Such designs suffer from fundamental limits that have been studied according to causality, related to dispersion relations, and theoretical limits of general physical systems. The root of these limits is associated with the well-known diffraction limit, in both spatial and angular domains.

*o.christogeorgos@qmul.ac.uk

$\dagger$ Also at School of Electronic and Optical Engineering, Nanjing University of Science and Technology, Nanjing 210094, China. haoyang.zhang@qmul.ac.uk

¥.cheng@qmul.ac.uk

§y.hao@qmul.ac.uk
Numerous efforts have been put forward to improve the performance by breaking the symmetry in the array geometry, notably, various optimization techniques have been proposed to achieve the "globally" optimal array often in random disordered distributions [3]. Furthermore, increasingly notable efforts have been made to apply disordered geometrical orders in the design of aperiodic arrays, by employing the use of aperiodic tilings, such as the Penrose tiling and Danzer tiling, as well as certain quasicrystals [4], or the sunflower seeds pattern [5] and even Fermat's spiral [6]. In addition, several efforts have been made to further optimize arrays that are based on aperiodic tilings so that the resulting arrays will meet a certain prescribed condition (e.g., peak side lobe level less than or equal to $-10 \mathrm{~dB}$ ) for a range of frequencies and beamsteering angles [7]. The proposed optimization techniques for arrays of radiation sources are in abundance in the existing literature. Several examples include the convex optimization technique [8-10], the matrix pencil method $[11,12]$, and the compressive sensing technique $[13,14]$.

The process of designing phased arrays with many elements and under certain spatial constraints (e.g., minimum element spacing, boundary conditions, etc.) is a highdimensional nonlinear optimization problem with many design variables. Evolutionary algorithms (EAs) have been used extensively in the past for the optimization of antenna arrays, due to their ability to handle such complex highdimensional problems. EAs are nature-inspired methods 
that mimic evolution mechanisms found in nature, in order to retrieve the optimal element arrangement that will lead to the desired array radiation characteristics. In many cases [15-20] some form of genetic algorithm (GA) is employed whose goal is to minimize an objective function through an iterative process. This process usually involves some sort of survival of the fittest procedure, where solutions that produce superior outputs survive in order to produce offspring (similar solutions) that will compete with others in the next generation (iteration), whereas weaker solutions die off. The procedure stops when a designer requirement is met (e.g., number of iterations, fitness function value, etc.) and the optimal solution that meets the prescribed performance requirements is found.

Antenna array optimization algorithms that are inspired by natural mechanisms are not limited only to GAs. In an effort to minimize the computational time and resources that are required to converge to the optimal solution, alternative nature-inspired EAs have been employed in the past. Specifically, there are several examples of natureinspired EAs that mimic animal, or even plant behavior, in order to converge to the optimal solution as fast as possible. Several examples include the invasive weed optimization, which is inspired by the phenomenon of colonization of invasive weeds in nature [21], the particle swarm optimization algorithm, which is a representation of the movement of organisms in a bird flock or fish school [22], the fruit-fly optimization algorithm, which is inspired by the stochastic behavior of fruit flies searching for food [23], the whale optimization algorithm, inspired by the humpback whales' hunting method [24], as well as the gray wolf optimizer, which mimics the social hierarchy and group hunting behavior of gray wolves in nature [25].

Most of the array optimization EAs that are found in the literature are used for very specific applications. These often include the application of the corresponding algorithm in order to design thinned or sparse arrays with a target specified radiation pattern. Another example is the design of arrays with nulls at certain angles in their radiation patterns (i.e., weak emission for a specified direction) or the design of arrays with as low side lobes as possible. In all of these cases, beginning from either a random or an ordered distribution, the goal of the optimization algorithm is to meet the design requirements with as few elements as possible in the shortest possible amount of time. This means that the faster the convergence of an algorithm, the better its performance for each specified application. The major drawback of the aforementioned optimization schemes lies in their inability to provide a globally optimal array element distribution that will operate with low peak side lobe level (PSLL) values for a range of frequencies and beam-steering angles. Instead, the above optimization algorithms provide the corresponding array element distributions that will meet the prescribed requirements only for the problem at hand (i.e., for fixed operating frequencies and beam-steering angles).

As mentioned earlier, there are some aperiodic element distributions that by breaking the symmetry in the array geometry manage to extend the array's operational bandwidth [4-7]. Although these element distributions manage to obtain high bandwidth ratios of even up to $22: 1$, they are not scalable. Thus, although these element distributions can be used for applications that require directive emission for a wide range of frequencies and beam-steering angles, they are limited to a relatively small amount of radiation sources due to the extensive computational time that would be required to optimize an extra-large array of thousands of elements. Therefore, it seems that there is yet to be found an array element distribution or optimization technique that will provide a global solution to the problem of designing wideband and steerable arrays of thousands of elements, under minimum element spacing constraints, without the need for huge computational times.

Here, we introduce a strategy for the design of phased arrays, which we view as a packing problem of a given number of emitters within a predefined space. The spatial optimization technique that is employed is very straightforward and easy to understand and the resulting element distributions are scalable, which can be used for the optimization of extra-large arrays of thousands of elements, consisting of optimized subarrays. The optimization is applied only to the subarray unit cell that can then be replicated along its axes, leading to a total structure that will maintain the prescribed radiation characteristics, thus saving huge amounts of computational time that would otherwise be required in order to optimize the whole structure. The resulting array element distribution adopts the properties of hyperuniform disordered systems and, as will be shown, is an element pattern scheme that is suitable for wideband directive phased arrays, under minimum element spacing constraints. Thus, in this work, we explore the hyperuniform disordered state of matter and its relation to the design of phased arrays that are characterized by directive emission for a broad frequency spectrum and large beam-steering angles near the horizon.

Disordered many-particle hyperuniform systems have already been found to have several applications in the fields of physics and materials science. Specifically, systems of disordered binary hard-sphere plasmas [26,27], as well as packings of maximally random jammed hard particles [28-30], are found to be hyperuniform. It is also observed that nonequilibrium phase transitions of manyparticle systems are accompanied by hyperuniform density fluctuations in the vicinity of the transition [31-34]. Hyperuniform disordered systems are also found in many natural and biological systems, such as the way that matter is distributed in the universe [35,36], as well as in the receptor 
organization patterns in the immune system [37] and the photoreceptor patterns on avian eye retina [38].

In addition, disordered hyperuniformity has led to major advances in the field of photonics. Because of their ability to control scattering properties, hyperuniform disordered structures have been used extensively to design complete photonic band gaps [39-43]. In Ref. [44] it was shown that materials made of scatterers distributed on a hyperuniform point pattern can be transparent for a broad range of frequencies and directions of incidence. Furthermore, Degl'Innocenti et al. [45] proposed and designed a hyperuniform disordered terahertz quantum cascade laser, whereas Castro-Lopez et al. [46] introduced hyperuniform disordered plasmonic gold surfaces with highly directive far-field diffraction patterns. Finally, the authors of Refs. [47,48] were able to design and manufacture hyperuniform disordered Luneburg lenses that possess peculiar properties, when compared to their metamaterial-based counterparts.

Here we demonstrate that the concept of disordered hyperuniformity warrants a solution to the packing problem that is associated with the array design procedure. In particular, due to the arrangement of the elements in real space, the array of radiation sources with hyperuniform disorder is characterized by several unique radiation properties in reciprocal space and provides an answer to the everlasting question that has been troubling antenna array designers and engineers for many decades: Given a specified maximum aperture size, what is an efficient way to distribute the array's radiation sources, subjecting to minimum element spacing constraints, in order to achieve directive emission, while being resistant to undesired interference, over a wide bandwidth and for large beam-steering angles?

\section{PRINCIPLE OF THE ARRAY WITH HYPERUNIFORM DISORDER}

A hyperuniform point distribution of $N$ points in $d$-dimensional Euclidean space $\mathbb{R}^{d}$ is one whose number variance $\sigma_{N}^{2}(R)$ within a spherical sampling window of radius $R$ increases at a rate that is slower than the window volume, i.e., slower than $R^{d}$, as the radius $R$ of the sampling window increases. This kind of point distribution is one in which (normalized) density fluctuations are completely suppressed at very large length scales [49], implying that its corresponding structure factor $S(\mathbf{k})$ will vanish in reciprocal space for small values of the wavevector $\mathbf{k}$. The structure factor, which is also referred to as the scattering pattern, is a physical quantity that gives information on how the geometrical arrangement of the particles inside a material affect the way the material scatters incident radiation. For a point configuration of $N$ points residing within a rectangular area of side lengths $L_{x}$ and $L_{y}$ at positions $\mathbf{r}_{1}, \mathbf{r}_{2}, \ldots, \mathbf{r}_{N}$, the structure factor is defined as

$$
S(\mathbf{k})=\frac{1}{N}\left|\sum_{j=1}^{N} e^{i \mathbf{k} \cdot \mathbf{r}_{j}}\right|^{2},
$$

where $\mathbf{k}$ is an appropriate infinite set of wavevectors. Specifically, we consider the computational domain to be a two-dimensional rectangular region in real space of side lengths $L_{x}$ and $L_{y}$, where periodic boundary conditions apply. Because of these conditions, the corresponding infinite set of wavevectors in reciprocal space is defined as

$$
\mathbf{k}=\left(\frac{2 \pi n_{x}}{L_{x}}, \frac{2 \pi n_{y}}{L_{y}}\right),
$$

where $n_{x}, n_{y} \in \mathbb{Z}$, indicating that the reciprocal space will be discretized when calculating the structure factor.

To generate a hyperuniform disordered point distribution, we follow the collective coordinate approach [50-52]. In particular, we generate a random point configuration that we aim to optimize to its ground state. This involves minimizing the total potential energy of the system until it reaches its absolute minimum, at or below a chosen cutoff in reciprocal space, while considering the sources to be hard disks of specified radius, thus introducing a minimum element spacing constraint. The total potential energy of the system is a function of both the position coordinates of the sources in real space, as well as the reciprocal space coordinates [50]. In our optimization algorithm (see Appendix A), we choose a value for the radius of the reciprocal space cutoff region $(0<|\mathbf{k}| \leq K)$ where the structure factor will vanish. Then, our algorithm generates the distribution that will meet the reciprocal space requirements, while taking into account the minimum element spacing constraint that is imposed. Note that the aforementioned constraint does not affect the hyperuniformity condition, since these two conditions have to be met simultaneously in order for our algorithm to converge. The resulting ground-state configuration will be transparent for the wavelengths that correspond to the wavevectors that lie within the exclusion region and, for this reason, it is termed stealthy [53].

Each resulting point pattern is described by the parameter $\chi=M(K) / 2 N \in[0,1]$, which is the ratio of the constrained degrees of freedom to the total number of degrees of freedom [see Table I of the Supplemental Material [54] for a tabulation of $K$ values and corresponding $M(K)$ values]. Here, parameter $\chi$ will be referred to as the stealthiness parameter. The greater the value of $\chi$, the more ordered the point distribution will be and, generally, if $\chi \leq 0.5$, the point distribution is considered to be in the disordered regime, but this threshold value varies depending on the number of points in the distribution. In particular, Uche et al. [50] conducted several simulations 


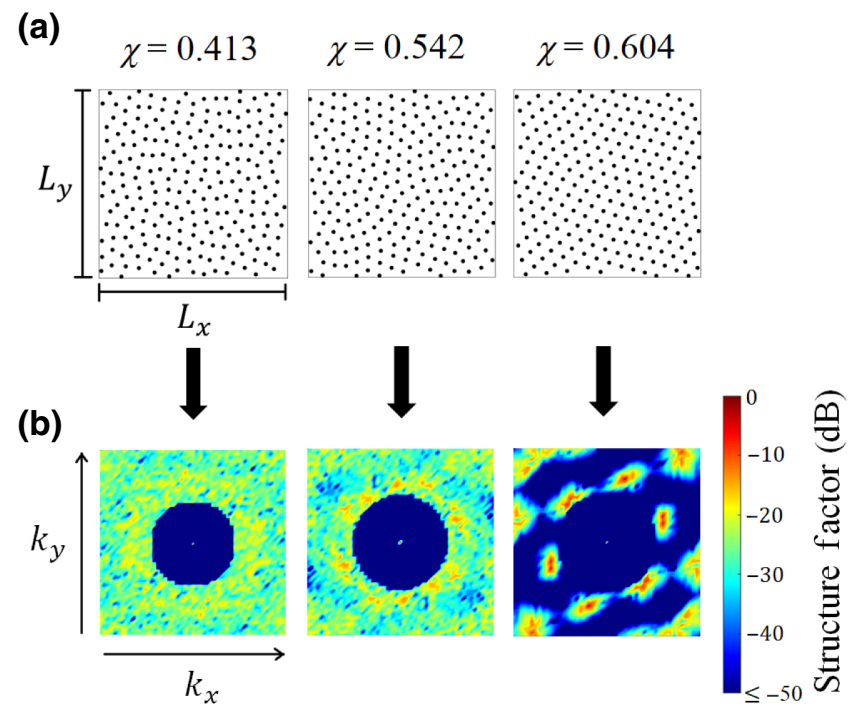

FIG. 1. The effect of the stealthiness parameter $(\chi)$ to the resulting real-space element locations (a) and the corresponding reciprocal space structure factor (b) for an array of 225 elements. The real-space computational domain is a rectangle of side lengths $L_{x}=L_{y}=1 \mathrm{~m}$. Both $k_{x}$ and $k_{y}$ reciprocal space coordinates span from $-50 \pi$ to $+50 \pi$. The structure factor is normalized to its maximum value and then plotted on a logarithmic scale.

for a varying number of points to determine this threshold value and came to the conclusion that, as the number of points increases, this value approaches a limit around $\chi=$ 0.58. In Fig. 1 we illustrate how the stealthiness parameter affects the resulting real-space element positions [Fig. 1(a)] and the corresponding reciprocal space structure factor [Fig. 1(b)] for an array with hyperuniform disorder of 225 elements. It can be easily seen that the greater the value of the stealthiness parameter, the larger the exclusion region in reciprocal space, but when $\chi$ is greater than its threshold value, the resulting configuration is crystalline and the circular exclusion region vanishes from the corresponding structure factor plot.

The problem of finding the hyperuniform distribution that corresponds to a particular $\chi$ value is essentially an eigenvalue problem. Specifically, the eigenvectors of the problem are the resulting hyperuniform distribution point coordinates and the eigenvalues are the $\chi$ values that correspond to each resulting distribution. As with all eigenvalue problems, the smaller the size of the system, the fewer uncorrelated eigenvalues that can be obtained. Therefore, the fewer points in a distribution, the fewer distinct $\chi$ values that can be obtained for the hyperuniform states.

\section{DESIGN AND IMPLEMENTATION}

The radiation pattern in reciprocal space of an array of identical elements can be described by its array factor. When the array factor is multiplied with the individual single antenna element radiation pattern, the result is the total radiation pattern of the array, without taking into account the mutual coupling between the elements. Therefore, its square norm can qualitatively describe the radiation pattern of a planar array of radiation sources, that is,

$$
|A(\mathbf{k})|^{2}=\left|\sum_{j=1}^{N} e^{i \mathbf{k} \cdot \mathbf{r}_{j}}\right|^{2},
$$

where $N$ identical elements are distributed in the $(x, y)$ plane of a Cartesian coordinate system $(x, y, z)$ at positions $\mathbf{r}_{1}, \mathbf{r}_{2}, \ldots, \mathbf{r}_{N}$. Here, the wavevector is associated with the operating wavelength $(\lambda)$ and the position of the observer in real space, which is described by the corresponding elevation and azimuth angles $(\theta, \phi)$ :

$$
\mathbf{k}=\frac{2 \pi}{\lambda} \sin \theta(\cos \phi, \sin \phi) .
$$

The differences between the physical meaning of the wavevectors that are defined in Eqs. (2) and (4) can be easily seen. The former is associated with the periodic boundary conditions that are imposed on the real-space unit cell and takes discrete values from $-\infty$ to $+\infty$, whereas the latter is associated with the position of the observer in real space and the operating wavelength and takes continuous values from $-2 \pi / \lambda$ to $+2 \pi / \lambda$. Apart from the aforementioned physical differences between the two wavevectors, the similarities between the normalized radiation pattern of an array of identical elements and the structure factor of a point distribution are readily apparent.

In order to determine what is the optimal $\chi$ value for an antenna array with hyperuniform disorder, we conducted an extensive parametric analysis for arrays with 16 up to 225 elements. In this parametric analysis, for each array with a fixed number of elements, at least ten different disordered hyperuniform array distributions with different $\chi$ parameter values are produced. During the analysis, for a fixed number of elements, we determined the $\chi$ values for which the results are satisfactory in terms of PSLL suppression for as wide a range of frequencies and steering angles as possible. For each number of elements, a lower and an upper bound for the $\chi$ value is determined for good performance according to the aforementioned performance requirements. The results of this analysis can be seen in Fig. 2. The optimal $\chi$ value for each number of elements resides within the shaded area of this graph and, for this reason, arrays with hyperuniform disorder (HUD) whose element distributions correspond to the $(N, \chi)$ combinations within the shaded area of Fig. 2 are considered to be optimal.

It is important to note here that, as the number of elements increases, the optimal $\chi$ bounds become more and more narrow and the optimal value tends to 0.5 , which is 


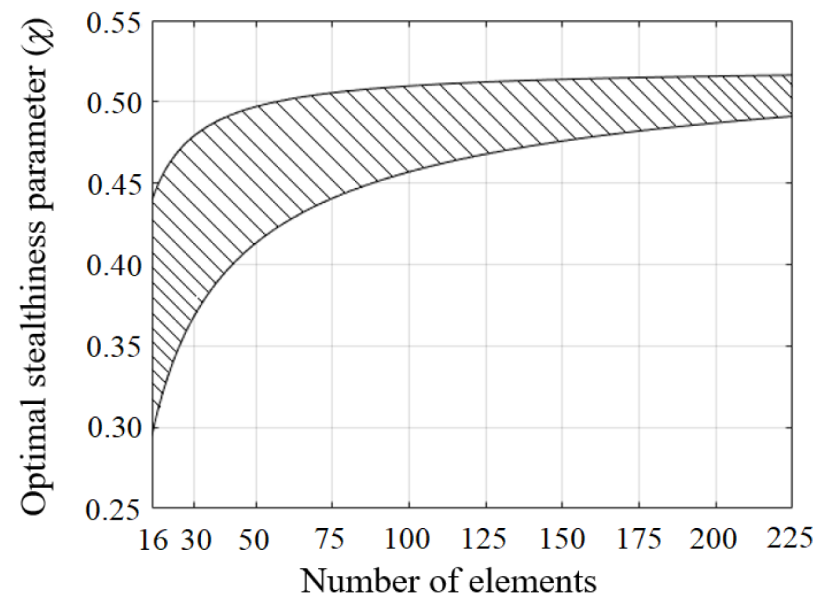

FIG. 2. The lower and upper bounds for the optimal value of the stealthiness parameter for an array with HUD for a varying number of elements. Arrays with $(N, \chi)$ combinations that reside within the shaded area will have good performance in terms of PSLL reduction for a wide operating bandwidth and large beamsteering angles.

in the middle of the disorder-order spectrum. On the other hand, for a low number of elements, the $\chi$ bounds for good performance are wider and this is due to the eigenvalue nature of the problem of generating hyperuniform distributions. As mentioned earlier, for a low number of array elements, the number of distinct $\chi$ values (eigenvalues) that can be obtained for the hyperuniform states are lower and, thus, the $\chi$ bounds for good performance in Fig. 2 are wider.

At this point, it is important to qualitatively review the radiation characteristics of the array with hyperuniform disorder by comparing its radiation pattern with other existing array element distributions. For this reason, in Fig. 3(a) we illustrate four different array element distributions, namely the $15 \times 15$ periodic array of 225 elements, the base Penrose-tiling array of 223 elements, the random array of 225 elements, and our proposed array of 225 elements with HUD and $\chi=0.489$. Note that the base Penrose-tiling array is generated by placing sources on the vertices of a thick-and-thin rhombus Penrose tiling. Although there are techniques that can be found in the existing literature to optimize certain arrays based on aperiodic tilings [7], here we use the base Penrose-tiling array where no optimization is applied, in order to compare our proposed design to not only periodic or random configurations, but to another state of matter, the quasicrystals. In Fig. 3 we illustrate the magnitude of the array factor, normalized to its maximum value and plotted in decibels. Note that the maximum value of the array factor is dependent only on the number of array elements and is thus exactly the same for any array element distribution with the same number of elements. In Fig. 3(b) the operating frequency is equal to $f_{0}$, with $f_{0}$ being the frequency for which the spacing between the sources of the $15 \times 15$ periodic array of 225 elements equals $\lambda_{0} / 2$, where $\lambda_{0}$ is the corresponding operating wavelength. The case in which $f=3 f_{0}$ is shown in Fig. 3(c) and a top-down view of Fig. 3(c) is shown in Fig. 3(d).

As expected, the periodic array, as well as the base Penrose-tiling array, suffer from strong grating lobes when $f=3 f_{0}$, which is not the case for either the random array or the array with hyperuniform disorder. Observing the radiation pattern of the array with hyperuniform disorder, one can see that the radiation is very weak within the circular region that surrounds the main lobe. Because of this, an incoming wave that is incident to the array from an angle that resides within this exclusion region would not alter the array's radiation pattern. Therefore, this circular exclusion region surrounding the main lobe of our proposed array element distribution acts as a protective layer of weak emission that surrounds the main beam and protects it from exterior interference. It is important to note here that, when calculating the structure factor, the distribution is considered to be replicated an infinite number of times along its axes and, for this reason, the structure factor values within the exclusion region are absolute zero [Fig. 1(b)]. On the other hand, during the array factor analysis the array is of finite dimensions. As a result, the finite effects of the square computational domain can be seen in the radiation pattern of the array with HUD, where the diffraction pattern of a square aperture can be seen within the exclusion region. Still though, the radiation values within the exclusion region are very low compared to the main lobe, as well as the peak side lobes, and so this region is termed a weak emission region.

Furthermore, the unique radiation characteristics of the array with hyperuniform disorder are maintained during beam steering, as evidenced in Fig. 3(e), which illustrates the top-down view of the radiation pattern polar plot when $f=3 f_{0}$ for all four array element distributions that are illustrated in Fig. 3(a) when the main lobe is steered towards the $\phi=0^{\circ}, \theta=30^{\circ}$ direction, measured from the array's bore sight. The weak emission exclusion region around the main lobe of the radiation pattern of the array with hyperuniform disorder is preserved during beam steering and it also acts as an indicator of the steering direction. This is in contrast with the random array's steered radiation pattern, where the absence of the circular exclusion region makes it very difficult to determine the steering direction with the naked eye. For the case of the periodic and base Penrose-tiling arrays, the presence of strong grating lobes makes it impossible to determine the steering direction.

From the above analysis, it can be easily seen that the periodic array has several exclusion regions in its radiation pattern, but suffers from strong grating lobes. On the other hand, the random array totally suppresses the grating lobes, but no exclusion regions are present in its 
(a)

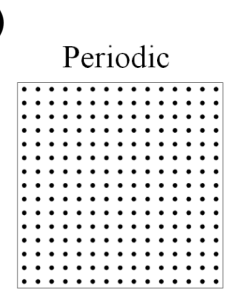

(b)

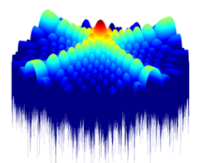

(c)

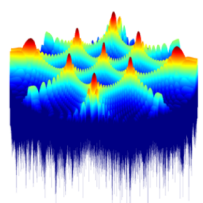

(d)

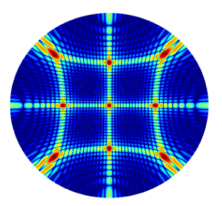

(e)

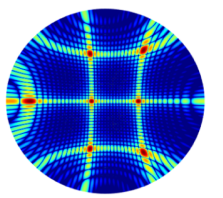

Base
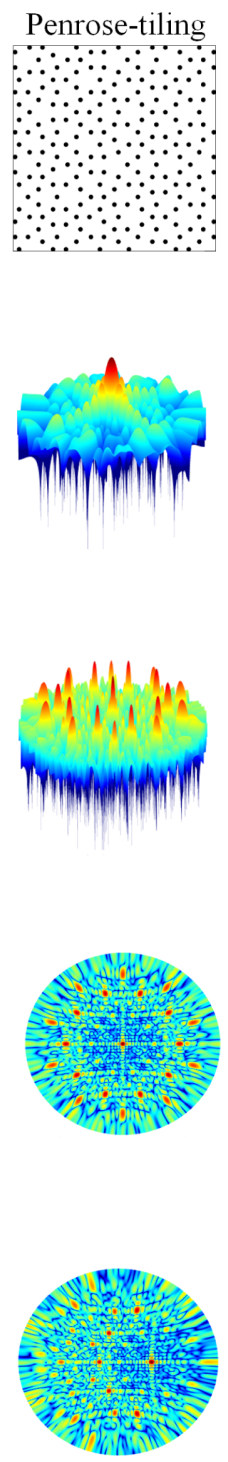
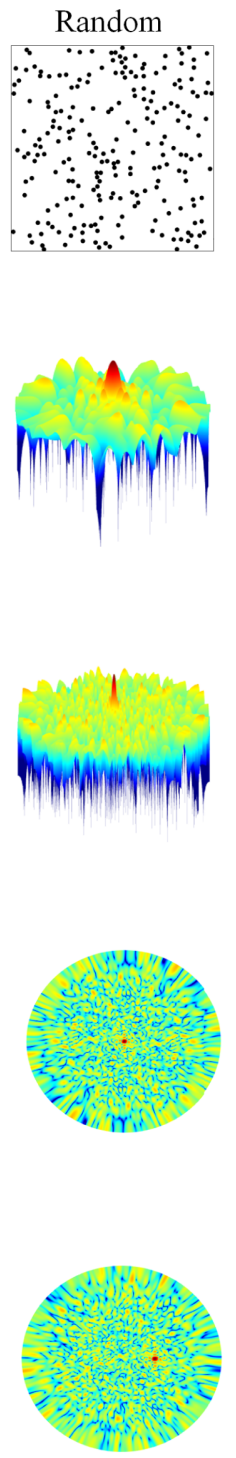

With HUD

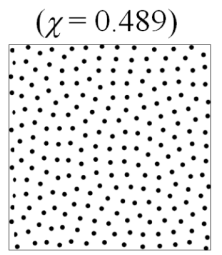

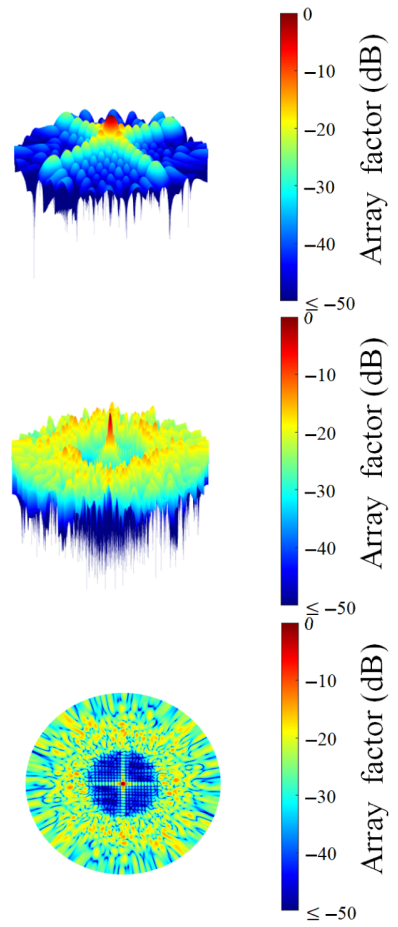

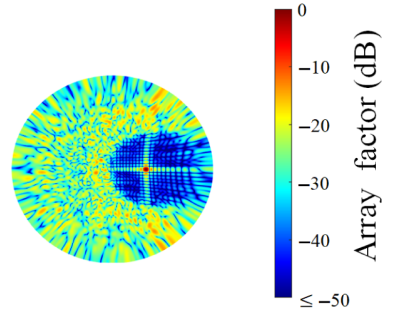

FIG. 3. The effect of the array element distribution on the radiation pattern. (a) The element distribution of the periodic array, the base Penrose-tiling array, the random array, and the array with hyperuniform disorder $(\chi=0.489)$. (b) The corresponding radiation pattern of the arrays when $f=f_{0}$ with $f_{0}$ being the frequency for which the distance between the sources of the periodic array equals one half of the corresponding operating wavelength. (c) The corresponding radiation pattern of the arrays when $f=3 f_{0}$. (d) The topdown view of the corresponding radiation pattern of the arrays when $f=3 f_{0}$. (e) The top-down view of the corresponding radiation pattern of the arrays when $f=3 f_{0}$ and the main beam is steered towards the $\phi=0^{\circ}, \theta=30^{\circ}$ direction, measured from the array's bore sight. In all panels the results from left to right correspond to the periodic array, the base Penrose-tiling array, the random array, and the array with hyperuniform disorder $(\chi=0.489)$. In the polar radiation plots the angular and radial coordinates correspond to the $\phi$ and $\theta$ angles, respectively.

corresponding radiation pattern. The array with hyperuniform disorder makes use of the best attributes from both states of matter (i.e., exclusion region zones and low PSLL values) and incorporates them into a single design, thus performing much better than both. The suppression of the grating lobes for a very wide range of frequencies makes the performance of our proposed design clearly better than the periodic or base Penrose-tiling element distributions. The clear advantage of our proposed array element distribution against its random counterpart is its ability to protect the main lobe of radiation from exterior interference, while maintaining low PSLL values and taking into account the minimum distance constraint between the radiation sources. 
It is worth noting here that, although the PSLL values remain low (see Fig. 1 of the Supplemental Material [54]), the size of the circular exclusion region in the radiation pattern of the array with HUD decreases as the operating frequency increases (see Fig. 2 of the Supplemental Material [54]), thus limiting the incident angles for which the main lobe will be protected from exterior interference. In particular, the size of the weak emission exclusion region in the radiation pattern of the array is determined by the structure factor cutoff radius $(K)$ that is selected when obtaining the hyperuniform disordered distribution, the operating wavelength $(\lambda)$, and the side length of the square array aperture $(L)$. Specifically, the radius of the weak emission region for a given operating wavelength and array aperture is given by the angle $\theta_{\mathrm{exc}}$ measured from the array's bore sight:

$$
\theta_{\mathrm{exc}}=\arcsin \left(\frac{\lambda}{L} \sqrt{K}\right)
$$

Inversely, for applications where the square aperture side length is given and we require that the exclusion region radius is equal to $\theta_{\text {exc }}$ in the array's radiation pattern for a given operating wavelength, then by using Eq. (5) we can extract the required cutoff radius $K$. This parameter can then be used to determine the number of required constrained degrees of freedom $M(K)$ (see Table I of the Supplemental Material [54]) and thus lead to the selection of the appropriate number of elements $(N)$ and $\chi$ parameter through the formula $\chi=M(K) / 2 N$. Here, attention should be given to ensure that the selected $(N, \chi)$ combination reside within the shaded area of Fig. 2, in order to ensure that the optimal array design is employed.

\section{EXPLORING THE CAUSALITY BEHIND THE UNIQUE RADIATION PATTERN}

In order to determine the reason behind the unique radiation pattern behavior of the array with hyperuniform disorder, we examine the site-to-site pair correlation between the sources in all of the array element distributions that are shown in Fig. 3(a). From statistical mechanics and particle physics, we know that the site-to-site pair correlation function $g_{2}(r)$, which is also termed the radial distribution function (RDF), is associated with the probability of finding a particle at a certain distance $r$ away from a reference particle. The RDF is calculated for each $r$ taking every particle as the reference point and then performing a mean average of the number of particles found at this distance. At the end, normalization is applied so that the RDF for each array element distribution can be compared to the RDF of an ideal gas (i.e., a totally random structure), where $g_{2}(r)=1$ for every $r$, meaning that the probability of finding a particle at a certain distance from a reference particle is uniform. Essentially, the RDF describes how-on average - the particles of a system are radially packed around each other.

In Fig. 4 we illustrate the results for the RDF of all the array element distributions that are shown in Fig. 3(a). Figures 4(a)-4(d) correspond to the cases of the periodic array, base Penrose-tiling array, random array, and array with HUD and $\chi=0.489$, respectively. A peak in the RDF corresponds to a nearest-neighbor distance. The RDF of a crystal structure, like the periodic array, is characterized by several peaks of high intensities. For the cases of the periodic and base Penrose-tiling arrays, the uniformity in their geometry leads to peaks of high intensities [note the vertical axis values in Figs. 4(a) and 4(b)], indicating that their first, second, third, etc. nearest neighbors are in fixed distances for all the points in the distribution. A random point distribution is devoid of any spatial uniformity and its RDF is relatively stable and equal to around unity for all the values of $r$. This RDF behavior corresponds to an ideal gas or liquid, where the particles are packed randomly together. For the case of the HUD distribution, a peak is observed for a low value of $r$ and after this value the RDF follows the ideal gas or liquid behavior. For this reason, a HUD distribution is said to have short-range crystal-like pair correlations and long-range liquidlike pair correlations.

To better understand the above, in Fig. 5 we illustrate how the points in the HUD distribution are packed around a specific reference point. Keep in mind that the behavior
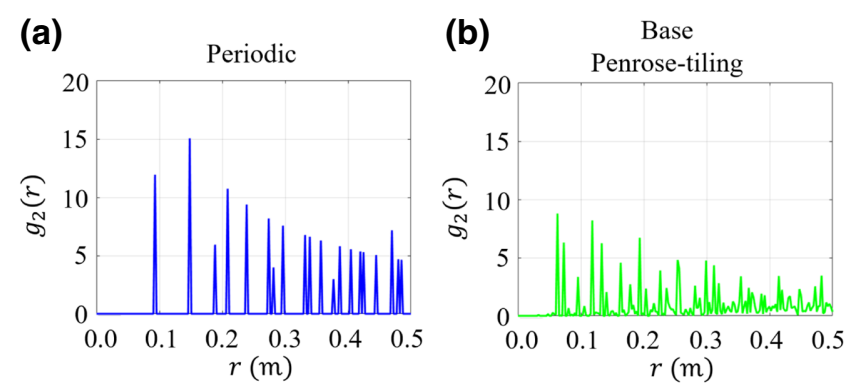

(c)

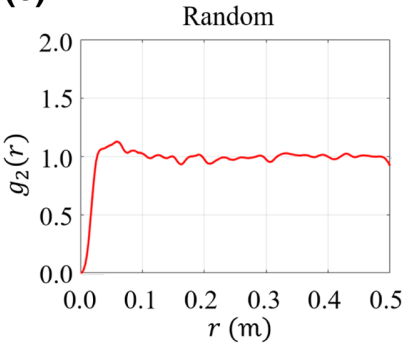

(d)

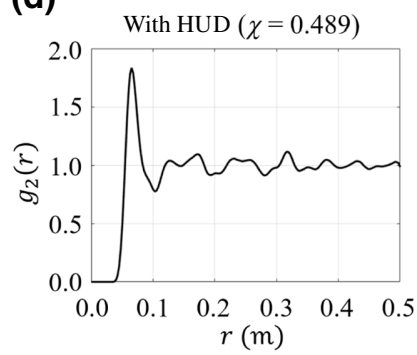

FIG. 4. Radial distribution function $g_{2}(r)$ for all the array element distributions that are shown in Fig. 3(a), namely the (a) periodic array, (b) base-Penrose tiling array, (c) random array, and (d) array with HUD and $\chi=0.489$. The distance parameter $r$ is in meters and takes values in the $[0, L / 2]$ interval, where $L$ is the side length of the square aperture area. 


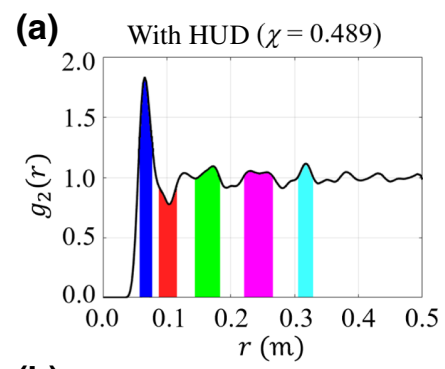

(b)

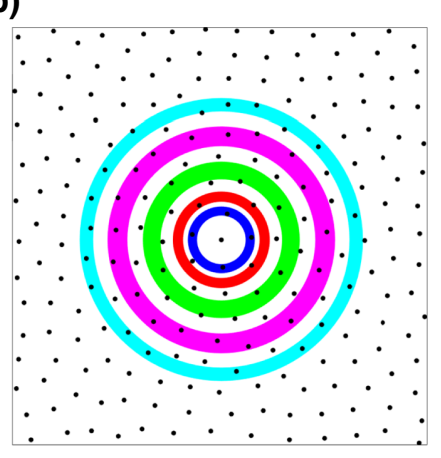

FIG. 5. (a) Radial distribution function $g_{2}(r)$ for the array with HUD and $\chi=0.489$ that is shown in Fig. 3(a). The distance parameter $r$ is in meters. (b) The distribution of points in the array with HUD and $\chi=0.489$ that is shown in Fig. 3(a), with the reference point in the centre. Around the reference point, several rings of different colors are drawn, whose radii are determined by the distance $r$ of the same colored regions in the RDF graph of (a).

of the RDF is associated with the probability of finding a number of points at a specific distance from a reference point, when all points in the distribution are taken as a reference while considering that the distribution is replicated an infinite number of times along its axes. Therefore, the observations that are made here are valid for all the points in the distribution and not only the reference point that is shown. In Fig. 5(a) we illustrate the RDF of the HUD distribution shown in Fig. 3(a) where some areas of the graph are colored. Fig 5(b) is a reillustration of the HUD distribution of Fig. 3(a), where several rings of different colors are drawn around the reference point in the center. The radii of the rings are determined by the distance $r$ of the corresponding colored parts in the RDF that is shown in Fig. 5(a). Note that the RDF is proportional to the number of points found at a distance $r$ from a reference point and inversely proportional to the distance $r$. Therefore, an accumulation of points at short range leads to high RDF values. In Fig. 5(b) it can be seen that there is an accumulation of points at a short range around the reference point (blue ring) that corresponds to the peak in the RDF of Fig. 5(a). As the distance from the reference point increases, the probability of finding a point at this distance lowers significantly, leading to the absence of points in the red ring of Fig. 5(b). After this distance, the RDF follows the behavior of a random process, thus totally suppressing long-range density fluctuations.

Because of its site-to-site pair correlation behavior, the array with hyperuniform disorder adopts the best attributes from each state of matter on the edges of the disorder-order spectrum. On the one hand, due to the crystal pair correlations between its sources, the periodic array has several exclusion regions in its radiation pattern that surround the undesired grating lobes. On the other hand, the radiation pattern of the totally random array is devoid of any grating lobes, as well as any exclusion regions. Because of the short-range crystal-like pair correlations between its radiation sources, the radiation pattern of the array with hyperuniform disorder is characterized by an exclusion region around the main lobe, while at the same time, due to its long-range liquidlike pair correlations, the grating lobes are completely suppressed and the side lobes are kept at a very low level for a wide range of frequencies, as well as beam-steering angles.

\section{SCALABILITY OF THE ARRAY WITH HYPERUNIFORM DISORDER}

Another feature of the array with hyperuniform disorder is its scalability. The two-dimensional computational domain can be considered to be a unit cell where Bloch's theorem with the use of periodic boundary conditions is applied. Therefore, periodically replicating a point distribution of locally hyperuniform disorder will lead to a global point distribution that is still hyperuniform disordered (see the supplemental material of Ref. [44] for a proof). To illustrate this attribute of the array with hyperuniform disorder, we used the array of 225 sources with hyperuniform disorder and $\chi=0.489$ as a subarray in a $3 \times 3$ arrangement leading to an array of 2025 radiation sources, as shown in Fig. 6(a).

In Figs. 6(b)-6(c) we illustrate the results for the aforementioned subarray arrangement. In Fig. 6(b) we illustrate four radiation pattern cuts for the subarray when the operating frequency equals $f=6 f_{0}$, where $f_{0}$ is the frequency for which the minimum spacing between the radiation sources of the subarray equals one half of the operating wavelength. Note that the subarray element distribution that is used is the same as that shown in Fig. 3(a). In Fig. 6(c) we illustrate the results for the large array with hyperuniform disorder made by the $3 \times 3$ subarray arrangement, where four radiation pattern cuts are plotted, with $f=6 f_{0}$ as well. As can be seen, the exclusion region property of the total array is preserved and the radiation pattern values within this region are much lower than the corresponding values of the subarray's radiation pattern. These results validate that the large array made by the $3 \times 3$ arrangement of a subarray with hyperuniform disorder is still hyperuniform disordered. If the element distribution is repeated an infinite number of times then 
(a)
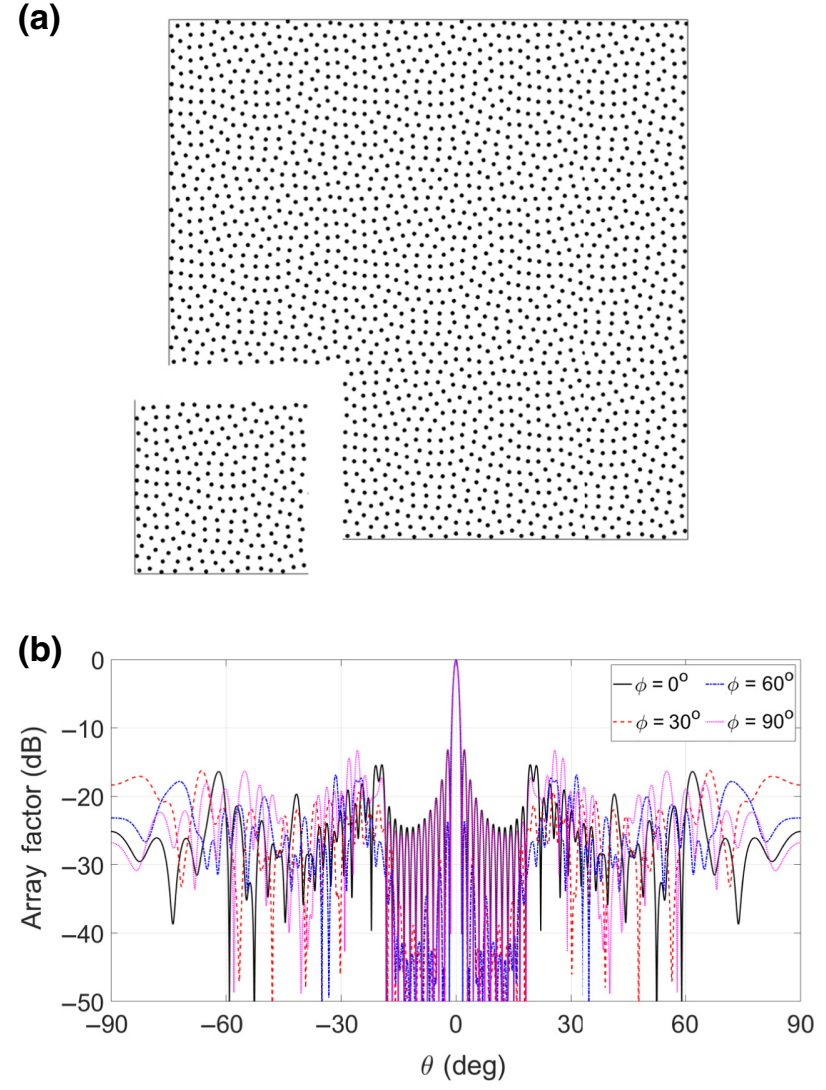

(c)

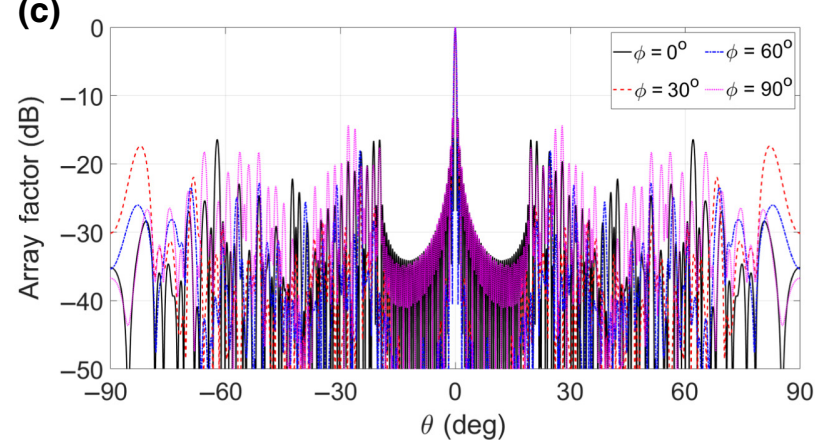

FIG. 6. Scalability of the array with hyperuniform disorder. (a) Large array element distribution made from the $3 \times 3$ arrangement of the subarray with HUD whose element distribution is shown cut off at the bottom left part of the figure for visualization purposes. Radiation pattern cuts of (b) the subarray and (c) the $3 \times 3$ large array that is shown in (a) when $f=$ $6 f_{0}$, where $f_{0}$ is the frequency for which the minimum distance between the radiation sources equals one half of the operating wavelength.

the radiation values within the exclusion region would be absolute zero, as indicated by the structure factor analysis in Fig. 1(b). Here, with only a $3 \times 3$ expansion of the original subarray we can already see that the nulling in the exclusion region is much deeper than the case of the single subarray. Note that the stealthiness parameter $\chi$ for the large array is the same as the array with HUD that is used as a subarray.

\section{PROOF-OF-CONCEPT DEMONSTRATION}

Here, we validate the above theoretical results with both simulation and measurements of an array fabricated for operation at microwave frequencies. Specifically, we fabricate a periodic array and an array with hyperuniform disorder and $\chi=0.438$ with 16 Vivaldi antennas as the individual elements for both array element distributions. The $\chi$ value here is chosen by observing Fig. 2 and generating arrays with 16 elements and $\chi$ values that reside within the shaded area of the graph. We then compare the performance of the generated arrays and choose the one with the best performance in terms of wideband side lobe suppression for wide scanning angles. For the fabricated arrays, we choose the Vivaldi antenna as the individual array element, due to its broadband behavior. Each Vivaldi antenna is designed and fabricated on a FR4 substrate with a relative permittivity of 4.3 , a loss tangent of 0.025 , and a thickness of $0.8 \mathrm{~mm}$. The dimensions of the antenna are optimized using CST Microwave Studio ${ }^{\circledR}$. Each antenna element is fed through a microstrip line and the overall antenna size of each element is $42 \times 44 \mathrm{~mm}^{2}$. In Fig. 7 we illustrate the fabricated 16-element Vivaldi antenna array with hyperuniform disorder and $\chi=0.438$ along with its test environment. Simulation results are obtained using CST, where the radiation patterns are calculated based on the full electromagnetic wave simulations of the arrays shown in Figs. 3(c) and 3(d) of the Supplemental Material [54]. For details on the dimensions of the elements

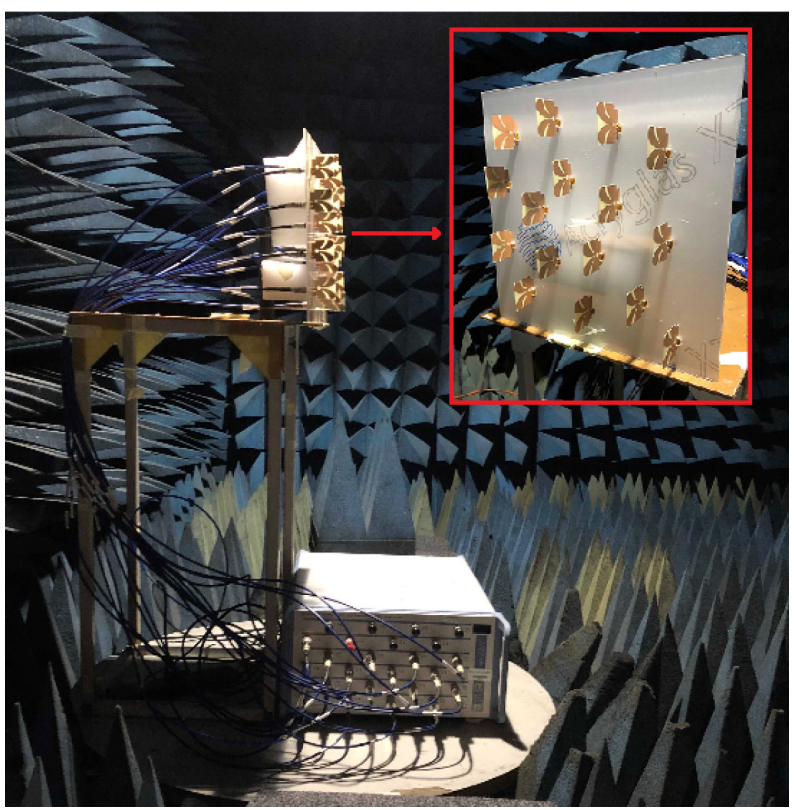

FIG. 7. The fabricated 16-element Vivaldi antenna array with hyperuniform disorder and $\chi=0.438$ and its test environment. 
(a)

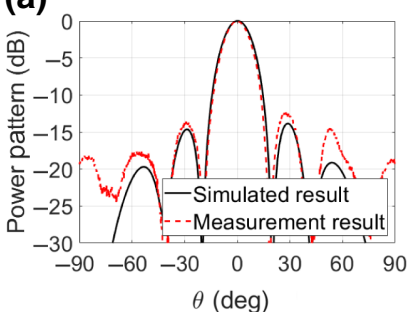

$\theta$ (deg)

(b)
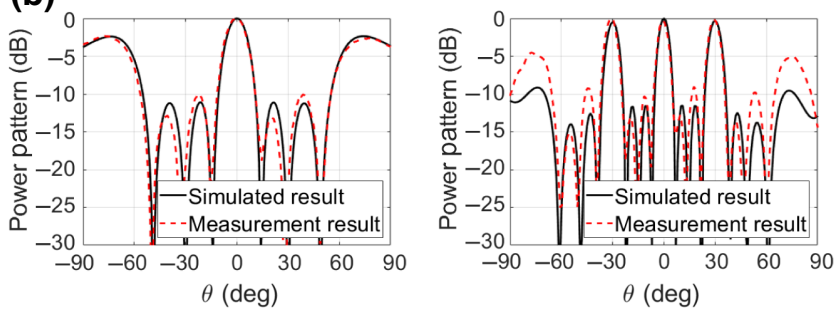

FIG. 8. Simulated (black solid line) and measured (red dotted line) radiation pattern results in the azimuth plane $\left(\phi=0^{\circ}\right)$ when $f=3 \mathrm{GHz}$ (left) and $f=6 \mathrm{GHz}$ (right) for (a) the 16-element Vivaldi antenna array with hyperuniform disorder and (b) the 16element Vivaldi periodic antenna array.

used, as well as the simulation and measurement setup of the arrays, see Ref. [54]. Interested readers are also referred to Ref. [55] for a sparse array imaging system implementation using the same simulation setup.

We conducted our experiments for two different operating frequencies, namely when $f=3$ and $6 \mathrm{GHz}$. The simulated and measured radiation patterns in the azimuth plane $\left(\phi=0^{\circ}\right)$ are shown in Fig. 8(a) for the array with hyperuniform disorder when $f=3 \mathrm{GHz}$ (left) and $f=$ $6 \mathrm{GHz}$ (right). The results for the periodic array for the same operating frequencies are given in Fig. 8(b). In both cases and for both operating frequencies, the measurement and simulation results agree well with each other in the main lobe region. The discrepancies between the measurement and simulation data outside the main lobe region are attributed mostly to the unavoidable misalignment of some of the antenna elements during our experiment. It can be clearly observed that, for both operating frequencies, the antenna array with hyperuniform disorder suppresses the grating lobes and has significantly reduced PSLL values compared to the periodic array. Specifically, simulated results at $3 \mathrm{GHz}$ indicate that the PSLL value is reduced from $-2.4 \mathrm{~dB}$ for the periodic array to $-13.85 \mathrm{~dB}$ for the array with hyperuniform disorder.

When the frequency is increased to $6 \mathrm{GHz}$, the average interelement spacing becomes larger than the $3 \mathrm{GHz}$ case, with regard to the operating wavelength and hence the PSLL values for both array element distributions increase. This can be observed from the simulated periodic array pattern where two strong grating lobes emerged alongside the main lobe. On the other hand, the array (a)
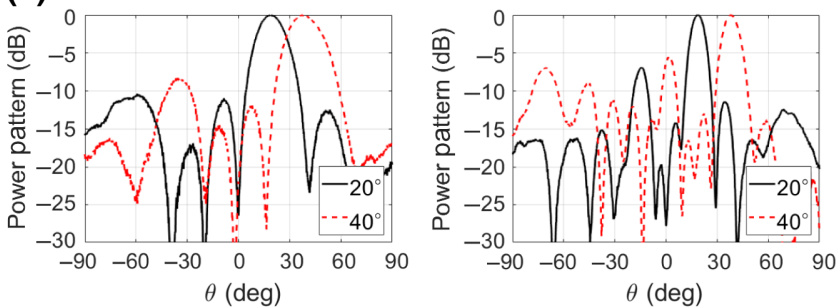

(b)
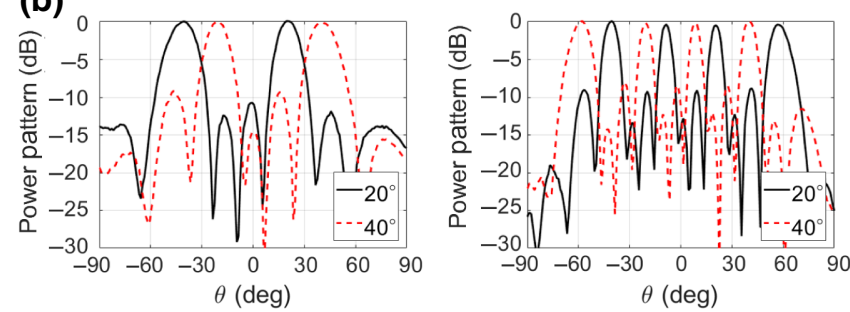

FIG. 9. Measured radiation pattern results in the azimuth plane $\left(\phi=0^{\circ}\right)$ when the main lobe is steered towards the $\phi=0^{\circ}, \theta=$ $20^{\circ}$ (black solid line) and the $\phi=0^{\circ}, \theta=40^{\circ}$ (red dotted line) directions when $f=3 \mathrm{GHz}$ (left) and $f=6 \mathrm{GHz}$ (right) for (a) the 16-element Vivaldi antenna array with hyperuniform disorder and (b) the 16-element Vivaldi periodic antenna array.

with hyperuniform disorder shows significantly suppressed grating lobes with a PSLL value of only $-11.5 \mathrm{~dB}$. The measured data match the simulation results fairly well in the vicinity of the main lobe and thus verify the accuracy of our simulations.

For each operating frequency, we also steered the main lobe towards two separate directions in the azimuth plane, namely the $\phi=0^{\circ}, \theta=20^{\circ}$ and the $\phi=0^{\circ}, \theta=$ $40^{\circ}$ directions. The measurement results in the azimuth plane $\left(\phi=0^{\circ}\right)$ are illustrated in Fig. 9(a) for the array with hyperuniform disorder and in Fig. 9(b) for the periodic array. As expected from our simulations, for both operating frequencies and steering directions, the array with hyperuniform disorder is characterized by a main beam of radiation that is surrounded by a weak emission exclusion region, whereas outside this exclusion region the side lobes are kept as low as possible, taking into account the fact that such a small number of elements would unavoidably lead to high PSLL values when large angle beam steering is applied and for high operating frequencies. The periodic array has several grating lobes that can be seen for both beam-steering directions and operating frequencies, just as our simulations predicted.

It should be noted that our measurement system is limited to 24 ports and, to ensure a fair comparison, we adopted a $4 \times 4$ element periodic array and a 16 element array with hyperuniform disorder. As mentioned earlier, due to the scalability of the array with hyperuniform disorder, the conclusions that are drawn from the measurement results in this section still apply to arrays with a larger 

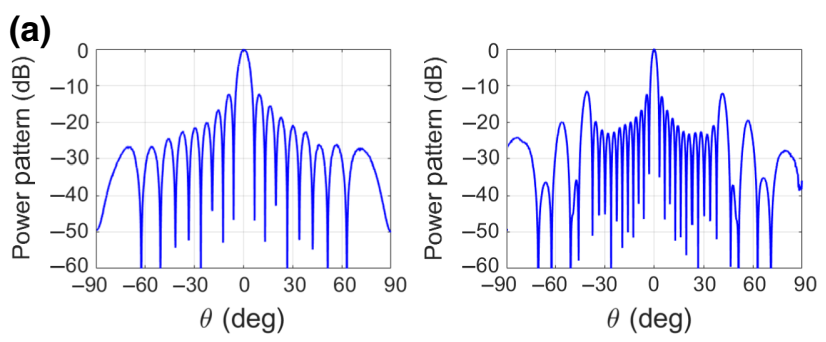

(b)
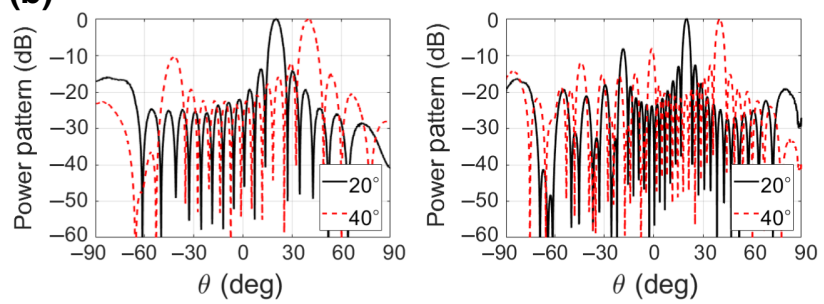

FIG. 10. Power pattern results in the azimuth plane $\left(\phi=0^{\circ}\right)$ for the large array with hyperuniform disorder made by the $3 \times 3$ arrangement of the fabricated subarray with hyperuniform disorder, shown in Fig. 7, when (a) no steering is applied and (b) when steering is applied towards the $\phi=0^{\circ}, \theta=20^{\circ}$ (black solid line) and $\phi=0^{\circ}, \theta=40^{\circ}$ (red dotted line) directions. The left-hand plots correspond to the case in which $f=3 \mathrm{GHz}$, whereas the right-hand plots correspond to the case in which $f=6 \mathrm{GHz}$.

number of elements. To illustrate this, we use the measurement results of this section to create a larger array made by the $3 \times 3$ subarray arrangement of the measured 16-element array with hyperuniform disorder. The resulting array configuration is made of 144 Vivaldi elements, which, as we have shown in the previous section, will be hyperuniform disordered as well. For detailed information regarding the coordinates of the elements in the 16-element Vivaldi subarray with hyperuniform disorder, see Table II of the Supplemental Material [54]. Since the subarray's unit cell side length equals $300 \mathrm{~mm}$, the element coordinates of the large array with hyperuniform disorder can easily be determined by shifting the element coordinates of the subarray with hyperuniform disorder by 0,300 , and $600 \mathrm{~mm}$ towards both $x$ and $y$ directions (totaling a $3 \times 3$ replica of the original subarray).

The measurement results of the 16-element array with hyperuniform disorder are used to extract the single Vivaldi antenna element radiation pattern in the HUD array configuration, while accounting for mutual coupling between the elements. Then multiplying this with the array factor of the large array leads to the results that are illustrated in Fig. 10. In order to ensure that this technique indeed takes into account the coupling between the elements, we multiplied the aforementioned single Vivaldi antenna element radiation pattern with the array factor of the subarray with hyperuniform disorder that is fabricated and the results are identical with the measured results of Fig. 8(a).
TABLE I. PSLL values (in decibels) derived from the power patterns illustrated in Fig. 10 for the large array of 144 Vivaldi elements made by the $3 \times 3$ arrangement of the fabricated subarray of 16 Vivaldi elements with hyperuniform disorder.

\begin{tabular}{ccc}
\hline \hline Frequency $(\mathrm{GHz})$ & Steering direction & PSLL $(\mathrm{dB})$ \\
\hline 3 & No steering & -12.29 \\
3 & $\phi=0^{\circ}, \theta=20^{\circ}$ & -13.6 \\
3 & $\phi=0^{\circ}, \theta=40^{\circ}$ & -10.38 \\
6 & No steering & -11.53 \\
6 & $\phi=0^{\circ}, \theta=20^{\circ}$ & -8.29 \\
6 & $\phi=0^{\circ}, \theta=40^{\circ}$ & -8.146 \\
\hline \hline
\end{tabular}

In Fig. 10(a) we show the case where no steering is applied, whereas in Fig. 10(b) we show the cases of beam steering towards the $\phi=0^{\circ}, \theta=20^{\circ}$ and $\phi=0^{\circ}, \theta=40^{\circ}$ directions. The left-hand plots correspond to when the operating frequency equals $3 \mathrm{GHz}$ and the right-hand plots correspond to an operating frequency of $6 \mathrm{GHz}$. As can be seen, for both operating frequencies and beam-steering directions, the large array of 144 Vivaldi elements made by the $3 \times 3$ arrangement of the fabricated subarray of 16 Vivaldi elements with hyperuniform disorder is still hyperuniform disordered, as the main beam is surrounded by the exclusion region. In Table I we provide the necessary information regarding the measured PSLL values for the radiation patterns that are shown in Fig. 10 of the large array with hyperuniform disorder. It is important to note here that the PSLL limit of $-10 \mathrm{~dB}$ is violated only when the operating frequency equals $6 \mathrm{GHz}$ and the main beam is steered towards the $\phi=0^{\circ}, \theta=40^{\circ}$ and $\theta=20^{\circ}$ directions, where the PSLL values equal -8.29 and $-8.146 \mathrm{~dB}$, respectively. These measurement results validate our claim that the array with hyperuniform disorder is indeed scalable and can be used for the design of large arrays of many elements without the need for huge computational times.

\section{DISCUSSION}

We have proposed and demonstrated how the concept of disordered hyperuniformity warrants an answer to the antenna array design, which we view as a packing problem. We have shown that the array whose elements are distributed according to a stealthy hyperuniform disordered point pattern has improved characteristics in terms of bandwidth and beam steering when compared to existing array element distributions. Specifically, in Ref. [56] an array of 256 elements was optimized by using an improved optimization algorithm to have PSLL values of less than $-10 \mathrm{~dB}$ for a $5: 1$ operating frequency range, whereas in Ref. [7] the authors employed their GA-inspired optimization algorithm to design an optimized Danzer array of 431 elements with PSLL values less than or equal to $-10 \mathrm{~dB}$ for a $22: 1$ operating frequency range. As evidenced in Fig. 1 of the Supplemental Material [54], our approach can 
lead to arrays of ultrawide bandwidth while taking into account the distance between the elements. In particular, our array with a HUD of 225 elements has low PSLL values of less than $-10 \mathrm{~dB}$ over at least a $60: 1$ operating bandwidth. Furthermore, the aforementioned optimization algorithms, as well as those that are reported in the existing literature, are inefficient for the optimization of extra-large arrays of thousands of elements, since the resulting array configurations are not scalable. A major advantage of our approach is the scalability of the resulting configurations, which can lead to the design of optimized extra-large arrays of thousands of elements without having to resort to the use of supercomputers.

A unique feature of arrays with HUD is the weak emission exclusion region that surrounds the main lobe of radiation. The very low values of radiation within this region guarantee that any incoming wave that is incident to the array with an angle less than $\theta_{\text {exc }}$ [Eq. (5)], measured from the array's bore sight, would have very low to almost zero effects on the array's performance, since the main lobe of radiation will be unaffected. Thus, the exclusion region acts as a protective layer that surrounds the main lobe and protects it from any exterior interference. Essentially, this means that an observer would see a main beam of radiation surrounded by an exclusion zone of weak radiation whose area gets smaller, compared to the wavevector space observation area, as the operating frequency increases (see Fig. 2 of the Supplemental Material [54]). This could be compensated by using Eq. (5) to determine the $(N, \chi)$ combination that would be required for an array with HUD for a fixed operating wavelength and aperture size, in order to obtain a radiation pattern whose exclusion region will reach the $\theta_{\text {exc }}$ angle that is desired.

In order to illustrate this, we designed three different arrays with HUD for three different exclusion region requirements. In particular, we designed three arrays with the same square aperture area of side length $L=0.5 \mathrm{~m}$ and set the operating frequency for all cases at $10 \mathrm{GHz}$. The requirement for the exclusion region radius is that, for a fixed operating frequency, each array should have $\theta_{\text {exc1 }}=$ $20^{\circ}, \theta_{\mathrm{exc} 2}=40^{\circ}$, and $\theta_{\mathrm{exc} 3}=60^{\circ}$, respectively. With a few calculations, from Eq. (5) we get $K_{1}=32, K_{2}=115$, and $K_{3}=208$ and, from Table I of the Supplemental Material [54], we have the corresponding required constrained degrees of freedom $M\left(K_{1}\right)=50, M\left(K_{2}\right)=178$, and $M\left(K_{3}\right)=332$. We observe that, for a fixed operating frequency, as the requirement for the size of the exclusion region increases, the required constrained degrees of freedom $M(K)$ increase as well. Since we require that the resulting number of elements and stealthiness parameter $(N, \chi)$ combinations reside within the shaded area of Fig. 2, the increase in the exclusion region size for a fixed operating frequency is translated into an increase in the array's required number of elements. The resulting $(N, \chi)$ combinations are given in the second column of Table II.
TABLE II. Required exclusion region size angles $\theta_{\text {exc }}$ (in degrees) at $10 \mathrm{GHz}$ and the corresponding number of elements and degree of stealthiness $(N, \chi)$ combinations for each case. The PSLL values are given in decibels and are calculated for the operating frequency of $10 \mathrm{GHz}$. Here $\Delta t$ corresponds to the computational time required (in minutes) for generating each array element distribution.

\begin{tabular}{cccc}
\hline \hline$\theta_{\text {exc }}(\mathrm{deg})$ & $(N, \chi)$ & PSLL $(\mathrm{dB})$ & $\Delta t(\min )$ \\
\hline 20 & $(55,0.455)$ & -5.801 & 1 \\
40 & $(180,0.494)$ & -10.02 & 62.4 \\
60 & $(330,0.503)$ & -13.18 & 705.9 \\
\hline \hline
\end{tabular}

In the same table, the PSLL values of each array at $10 \mathrm{GHz}$ and the computational time required for generating each array element distribution are given as well. It can be seen that, as the number of elements increases, the PSLL values decrease significantly, whereas the computational time required to generate the hyperuniform disordered distribution increases rapidly. The resulting element distributions and radiation patterns can be seen in Fig. 4 of the Supplemental Material [54].

The use of hyperuniform disordered element distributions in the field of phased arrays could be seen as a natural array optimization technique, in the sense that this kind of distribution is inspired by spatial distributions that can be found in many natural, biological, and physical systems. Here, we focus our interest on understanding the physics behind the use of HUD systems in the field of phased antenna arrays for ultrawide band and large beam-steering angle side lobe suppression, while taking into account the physical dimensions of the array's elements. The resulting array configurations with HUD are scalable and can be employed for the optimization of arrays of thousands of elements without needing to resort to the use of supercomputers to decrease the huge computational times that would be required when using existing array optimization techniques. The array with hyperuniform disorder adopts the best characteristics from both states of matter on the edges of the disorder-order spectrum. Because of its short-range crystal-like pair correlations, a weak radiation exclusion region surrounds the main lobe and protects it from any undesired interference, whereas, due to its long-range liquidlike pair correlations, the grating lobes are completely suppressed and the side lobes are kept at very low levels. For this reason, we consider the hyperuniform disordered element distribution to be an efficient answer to the packing problem that is intrinsic to the antenna array design procedure, in a manner that the available space is used as efficiently as possible to achieve high-quality wideband behavior that is preserved during beam steering.

It is worth noting here that, for a specific number of elements, infinite point distributions can be generated within the same aperture that will have the same $\chi$ values. Therefore, the use of hyperuniform disordered element 
distribution in the field of phased antenna arrays can provide infinite solutions to a highly nonlinear problem with many variables, through a deterministic procedure (minimization of the distribution's potential energy within the cutoff region in reciprocal space). Because of its unique site-to-site pair correlation behavior, the proposed element distribution manages to suppress the grating lobes, like a random array, while maintaining an exclusion region of weak radiation around the main lobe, like a periodic array. Furthermore, the array with hyperuniform disorder maintains its radiation characteristics during beam steering and most importantly for an ultrawide range of frequencies as the number of elements increases. Finally, the scalability of the proposed disordered array element distribution can be exploited in order to design extra-large arrays of many thousands of elements by replicating as many times as required the subarray with hyperuniform disorder, without the need for optimizing the total array.

Our work has therefore led to a potential paradigm shift in the classic theory of array designs, which is largely based on the diffraction theory of wave physics leading to the fundamental limit of radiation bandwidth and efficiency. By linking the element distribution disorder and especially treating the array design as a packing problem, we have proposed an array element distribution that is hyperuniform disordered and exhibits directive radiation for large beam-steering angles and wide operating bandwidths without exhausting computing power for blind optimization processes. This work adds to the existing research that has been conducted where the concept of reciprocal lattice or reciprocal space is introduced to explain the behavior of solids. Here, this is further explored to study antenna or laser arrays for bespoke emission and beam-steering applications by exploiting the extraordinary scattering properties of hyperuniform disordered systems.

\section{ACKNOWLEDGMENTS}

Y.H. initiated the idea and supervised the work. O.C. and Y.H. developed the theoretical work. H.Z. designed and simulated the hyperuniform antenna array. Q.C. and O.C. conducted the experiments and analyzed the data. O.C. and H.Z. contributed equally to this work. All authors have contributed to the manuscript preparation. Y.H. would like to thank the Engineering and Physical Sciences Research Council (EPSRC), UK for funding this research under Grants No. EP/S010009/1, No. EP/R035393/1, and No. EP/N010493/1 and the IET for the AF Harvey Research Prize. H.Z.'s work is supported by the China Scholarship Council under Grant No. 201706840031. O.C. would like to thank Mr. George Sakellion (Chem. M.Eng.) for helpful discussions on particle physics topics.

\section{APPENDIX A: ALGORITHM FOR GENERATING A STEALTHY HYPERUNIFORM DISORDERED DISTRIBUTION OF IMPENETRABLE DISKS}

The first step in our optimization algorithm is to choose the number of points $N$, the side lengths of the computational domain $L_{x}, L_{y}$, and the cutoff region radius $K$ where the structure factor will vanish, so that $\chi<0.58$ if $N>100$, or $\chi<0.5$ if $N \leq 100$, in order for the resulting configuration to belong to the disordered regime.

In our algorithm two real-space constraints are imposed. The first one is the minimum element spacing constraint, which is imposed by considering the sources as impenetrable hard disks of radius $R$. The second constraint that is applied is that all the hard disks should reside in their entirety within the computational domain of interest. Following the formulation that is presented in Ref. [50], we work with the real quantities

$$
\begin{aligned}
C(\mathbf{k}) & =\sum_{j=1}^{N-1} \sum_{l=j+1}^{N} \cos \left[\mathbf{k} \cdot\left(\mathbf{r}_{j}-\mathbf{r}_{l}\right)\right] \\
& =N \frac{S(\mathbf{k})-1}{2}
\end{aligned}
$$

where $\mathbf{r}_{j}$ is the position vector of the $j$ th point, $S(\mathbf{k})$ is the structure factor, and $\mathbf{k}$ is a properly defined infinite set of wavevectors as defined in Eq. (2).

Our objective is to find the point distribution that will minimize the total potential energy of the system under the spatial constraints that are imposed. The total potential energy of the system can be written as

$$
\Phi=\frac{1}{L_{x} L_{y}} \sum_{\mathbf{k}} C(\mathbf{k}),
$$

where $\mathbf{k}$ should be chosen so that the cutoff region of radius $K$ will be in its entirety within the reciprocal space defined by $\mathbf{k}$. The number of wavevectors that will reside within the exclusion region is denoted by $2 M(K)+1$, where the +1 corresponds to the origin in reciprocal space $(|\mathbf{k}|=0)$. Then, the absolute minimum of the total potential energy that we seek to obtain is equal to $\Phi_{\min }=$ $(N / 2)[1-2 M(K)]$. In our algorithm, we minimize $\Phi$ for every $|\mathbf{k}| \in K^{*}$ and therefore the goal for the absolute minimum of the potential energy is $\Phi_{\min }=(N / 2)[-2 M(K)]$. Note that, as the number of constrained degrees of freedom $M(K)$ increases, the value of the absolute minimum of the energy function $\Phi$ gets smaller, when the number of points remain constant.

Of course, the number of the constrained degrees of freedom $M(K)$ is dependent on the parameter $K$. Essentially, $K$ is the radius of the circle in which $S(\mathbf{k}) \rightarrow 0$ in reciprocal space. Outside of this circle the $S(\mathbf{k})$ values are considered as "wild" (i.e., random values greater than zero and 
much smaller than the value at the origin). Several values for the cutoff radius $K$ and the corresponding $M(K)$ values are given in Table I of the Supplemental Material [54]. Following the formulation of Ref. [50], the cutoff radius $K$ is equal to the square of the reduced distances $\left|k_{i}\right| /\left|k_{1}\right|$, where $\left|k_{1}\right|=2 \pi / L$ for the square lattice of $\mathbf{k}$ vectors, with $L_{x}=L_{y}=L$. Therefore, in our algorithm, the constraint for $|\mathbf{k}|$ being within the cutoff disk of radius $K$ in reciprocal space is defined as

$$
\begin{aligned}
& \left(\frac{k_{x}}{k_{1}}\right)^{2}+\left(\frac{k_{y}}{k_{1}}\right)^{2} \leq\left(\frac{k_{i}}{k_{1}}\right)^{2} \\
& \Longrightarrow\left(\frac{2 \pi n_{x} / L}{2 \pi / L}\right)^{2}+\left(\frac{2 \pi n_{y} / L}{2 \pi / L}\right)^{2} \leq K \\
& \Longrightarrow n_{x}^{2}+n_{y}^{2} \leq K .
\end{aligned}
$$

We implement the above using the fmincon function of MATLAB $^{\circledR}$ and the interior-point algorithm to find the point distribution that will minimize the total potential energy of the system under the aforementioned constraints.

\section{APPENDIX B: THE STEERED RADIATION PATTERN}

In order to steer the main lobe towards a specific direction in real space, we have to multiply the signal from every element in the array with a complex phase $\left(w_{j}\right)$, where $j \in[1, N]$, with $N$ being the number of elements in the array. Then, when the signals from each element are added together to form the output of the array, they would combine coherently. This process is called beam steering. If we desire the main lobe to be steered towards the elevation angle $\theta_{d}$ and the azimuth angle $\phi_{d}$, then the complex phase weight applied to the $j$ th array element will be defined as

$$
w_{j}=e^{-i \mathbf{k}_{d} \cdot \mathbf{r}_{j}},
$$

where $\mathbf{r}_{j}$ is the position vector of the $j$ th array element and $\mathbf{k}_{d}$ is the steered wavevector defined as

$$
\mathbf{k}_{d}=\frac{2 \pi}{\lambda} \sin \theta_{d}\left(\cos \phi_{d}, \sin \phi_{d}\right)
$$

with $\lambda$ being the operating wavelength. Therefore, taking into account beam steering, the normalized radiation pattern can be written as

$$
R\left(\mathbf{k}_{t}\right)=\left|\sum_{j=1}^{N} e^{-i \mathbf{k}_{d} \cdot \mathbf{r}_{j}} e^{i \mathbf{k} \cdot \mathbf{r}_{j}}\right|^{2}=\left|\sum_{j=1}^{N} e^{i \mathbf{k}_{t} \cdot \mathbf{r}_{j}}\right|^{2},
$$

where $\mathbf{k}$ is the wavevector associated with the observer position and the operating wavelength and $\mathbf{k}_{t}=\mathbf{k}-\mathbf{k}_{d}$ is the total wavevector when taking into account beam steering and the observer's position. By observing Eq. (B3), it can be easily seen that the steered radiation pattern formula is still very similar to the structure factor formula of Eq. (1) and the radiation pattern will maintain its characteristics during beam steering, with the only addition that the wavevector will now also depend on the steering direction.

[1] G. Y. Slepyan and A. Boag, Quantum Nonreciprocity of Nanoscale Antenna Arrays in Timed Dicke States, Phys. Rev. Lett. 111, 023602 (2013).

[2] M. P. Fischer, C. Schmidt, E. Sakat, J. Stock, A. Samarelli, J. Frigerio, M. Ortolani, D. J. Paul, G. Isella, and A. Leitenstorfer et al., Optical Activation of Germanium Plasmonic Antennas in the Mid-Infrared, Phys. Rev. Lett. 117, 047401 (2016).

[3] A. El-Makadema, L. Rashid, and A. K. Brown, Geometry design optimization of large-scale broadband antenna array systems, IEEE Trans. Antennas Propag. 62, 1673 (2013).

[4] V. Pierro, V. Galdi, G. Castaldi, I. M. Pinto, and L. B. Felsen, Radiation properties of planar antenna arrays based on certain categories of aperiodic tilings, IEEE Trans. Antennas Propag. 53, 635 (2005).

[5] M. C. Viganó, G. Toso, G. Caille, C. Mangenot, and I. E. Lager, Sunflower array antenna with adjustable density taper, Int. J. Antennas Propag. 2009 (2009).

[6] L. H. Gabrielli and H. E. Hernandez-Figueroa, Aperiodic antenna array for secondary lobe suppression, IEEE Photon. Technol. Lett. 28, 209 (2015).

[7] T. G. Spence and D. H. Werner, Design of broadband planar arrays based on the optimization of aperiodic tilings, IEEE Trans. Antennas Propag. 56, 76 (2008).

[8] B. Fuchs, Synthesis of sparse arrays with focused or shaped beampattern via sequential convex optimizations, IEEE Trans. Antennas Propag. 60, 3499 (2012).

[9] M. D’Urso, G. Prisco, and R. M. Tumolo, Maximally sparse, steerable, and nonsuperdirective array antennas via convex optimizations, IEEE Trans. Antennas Propag. 64, 3840 (2016).

[10] P. You, Y. Liu, S.-L. Chen, K. Da Xu, W. Li, and Q. H. Liu, Synthesis of unequally spaced linear antenna arrays with minimum element spacing constraint by alternating convex optimization, IEEE Antennas Wirel. Propag. Lett. 16, 3126 (2017).

[11] Y. Liu, Q. H. Liu, and Z. Nie, Reducing the number of elements in the synthesis of shaped-beam patterns by the forward-backward matrix pencil method, IEEE Trans. Antennas Propag. 58, 604 (2009).

[12] H. Shen and B. Wang, Two-dimensional unitary matrix pencil method for synthesizing sparse planar arrays, Digit. Signal Process. 73, 40 (2018).

[13] F. Viani, G. Oliveri, and A. Massa, Compressive sensing pattern matching techniques for synthesizing planar sparse arrays, IEEE Trans. Antennas Propag. 61, 4577 (2013).

[14] F. Yan, P. Yang, F. Yang, and T. Dong, Synthesis of planar sparse arrays by perturbed compressive sampling framework, IET Microwaves, Antennas Propag. 10, 1146 (2016). 
[15] R. L. Haupt, Thinned arrays using genetic algorithms, IEEE Trans. Antennas Propag. 42, 993 (1994).

[16] M. G. Bray, D. H. Werner, D. W. Boeringer, and D. W. Machuga, Optimization of thinned aperiodic linear phased arrays using genetic algorithms to reduce grating lobes during scanning, IEEE Trans. Antennas Propag. 50, 1732 (2002).

[17] S. Caorsi, A. Lommi, A. Massa, and M. Pastorino, Peak sidelobe level reduction with a hybrid approach based on gas and difference sets, IEEE Trans. Antennas Propag. 52, 1116 (2004).

[18] J. S. Petko and D. H. Werner, The evolution of optimal linear polyfractal arrays using genetic algorithms, IEEE Trans. Antennas Propag. 53, 3604 (2005).

[19] J. N. Bogard and D. H. Werner, in IEEE International Radar Conference, 2005. (IEEE, Arlington, VA, 2005), p. 905.

[20] R. L. Haupt, Optimized element spacing for low sidelobe concentric ring arrays, IEEE Trans. Antennas Propag. 56, 266 (2008).

[21] S. Karimkashi and A. A. Kishk, Invasive weed optimization and its features in electromagnetics, IEEE Trans. Antennas Propag. 58, 1269 (2010).

[22] R. Bhattacharya, T. K. Bhattacharyya, and R. Garg, Position mutated hierarchical particle swarm optimization and its application in synthesis of unequally spaced antenna arrays, IEEE Trans. Antennas Propag. 60, 3174 (2012).

[23] A. Darvish and A. Ebrahimzadeh, Improved fruit-fly optimization algorithm and its applications in antenna arrays synthesis, IEEE Trans. Antennas Propag. 66, 1756 (2018).

[24] C. Zhang, X. Fu, L. P. Ligthart, S. Peng, and M. Xie, Synthesis of broadside linear aperiodic arrays with sidelobe suppression and null steering using whale optimization algorithm, IEEE Antennas Wirel. Propag. Lett. 17, 347 (2018).

[25] X. Li and K. M. Luk, The grey wolf optimizer and its applications in electromagnetics, IEEE Trans. Antennas Propag. 68, 2186 (2019).

[26] E. Lomba, J.-J. Weis, and S. Torquato, Disordered hyperuniformity in two-component nonadditive hard-disk plasmas, Phys. Rev. E 96, 062126 (2017).

[27] E. Lomba, J.-J. Weis, and S. Torquato, Disordered multihyperuniformity derived from binary plasmas, Phys. Rev. E 97, 010102 (2018).

[28] A. Donev, F. H. Stillinger, and S. Torquato, Unexpected Density Fluctuations in Jammed Disordered Sphere Packings, Phys. Rev. Lett. 95, 090604 (2005).

[29] M. Skoge, A. Donev, F. H. Stillinger, and S. Torquato, Packing hyperspheres in high-dimensional euclidean spaces, Phys. Rev. E 74, 041127 (2006).

[30] C. E. Zachary, Y. Jiao, and S. Torquato, Hyperuniform Long-Range Correlations are a Signature of Disordered Jammed Hard-Particle Packings, Phys. Rev. Lett. 106, 178001 (2011).

[31] R. L. Jack, I. R. Thompson, and P. Sollich, Hyperuniformity and Phase Separation in Biased Ensembles of Trajectories for Diffusive Systems, Phys. Rev. Lett. 114, 060601 (2015).

[32] D. Hexner and D. Levine, Hyperuniformity of Critical Absorbing States, Phys. Rev. Lett. 114, 110602 (2015).
[33] E. Tjhung and L. Berthier, Hyperuniform Density Fluctuations and Diverging Dynamic Correlations in Periodically Driven Colloidal Suspensions, Phys. Rev. Lett. 114, 148301 (2015).

[34] D. Hexner and D. Levine, Noise, Diffusion, and Hyperuniformity, Phys. Rev. Lett. 118, 020601 (2017).

[35] A. Gabrielli, M. Joyce, and F. S. Labini, Glass-like universe: Real-space correlation properties of standard cosmological models, Phys. Rev. D 65, 083523 (2002).

[36] A. Gabrielli, B. Jancovici, M. Joyce, J. Lebowitz, L. Pietronero, and F. S. Labini, Generation of primordial cosmological perturbations from statistical mechanical models, Phys. Rev. D 67, 043506 (2003).

[37] A. Mayer, V. Balasubramanian, T. Mora, and A. M. Walczak, How a well-adapted immune system is organized, Proc. Natl. Acad. Sci. 112, 5950 (2015).

[38] Y. Jiao, T. Lau, H. Hatzikirou, M. Meyer-Hermann, J. C. Corbo, and S. Torquato, Avian photoreceptor patterns represent a disordered hyperuniform solution to a multiscale packing problem, Phys. Rev. E 89, 022721 (2014).

[39] M. Florescu, S. Torquato, and P. J. Steinhardt, Complete band gaps in two-dimensional photonic quasicrystals, Phys. Rev. B 80, 155112 (2009).

[40] S. Imagawa, K. Edagawa, K. Morita, T. Niino, Y. Kagawa, and M. Notomi, Photonic band-gap formation, light diffusion, and localization in photonic amorphous diamond structures, Phys. Rev. B 82, 115116 (2010).

[41] W. Man, M. Florescu, K. Matsuyama, P. Yadak, G. Nahal, S. Hashemizad, E. Williamson, P. Steinhardt, S. Torquato, and P. Chaikin, Photonic band gap in isotropic hyperuniform disordered solids with low dielectric contrast, Opt. Express 21, 19972 (2013).

[42] W. Man, M. Florescu, E. P. Williamson, Y. He, S. R. Hashemizad, B. Y. Leung, D. R. Liner, S. Torquato, P. M. Chaikin, and P. J. Steinhardt, Isotropic band gaps and freeform waveguides observed in hyperuniform disordered photonic solids, Proc. Natl. Academy Sci. 110, 15886 (2013).

[43] T. Amoah and M. Florescu, High-q optical cavities in hyperuniform disordered materials, Phys. Rev. B 91, 020201 (2015).

[44] O. Leseur, R. Pierrat, and R. Carminati, High-density hyperuniform materials can be transparent, Optica 3, 763 (2016).

[45] R. Degl'Innocenti, Y. Shah, L. Masini, A. Ronzani, A. Pitanti, Y. Ren, D. Jessop, A. Tredicucci, H. E. Beere, and D. A. Ritchie, Hyperuniform disordered terahertz quantum cascade laser, Sci. Rep. 6, 19325 (2016).

[46] M. Castro-Lopez, M. Gaio, S. Sellers, G. Gkantzounis, M. Florescu, and R. Sapienza, Reciprocal space engineering with hyperuniform gold disordered surfaces, APL Photonics 2, 061302 (2017).

[47] B.-Y. Wu, X.-Q. Sheng, and Y. Hao, Effective media properties of hyperuniform disordered composite materials, PLoS One 12, e0185921 (2017).

[48] H. Zhang, H. Chu, H. Giddens, W. Wu, and Y. Hao, Experimental demonstration of luneburg lens based on hyperuniform disordered media, Appl. Phys. Lett. 114, 053507 (2019).

[49] S. Torquato, Disordered hyperuniform heterogeneous materials, J. Phys.: Condens. Matter 28, 414012 (2016). 
[50] O. U. Uche, F. H. Stillinger, and S. Torquato, Constraints on collective density variables: Two dimensions, Phys. Rev. E 70, 046122 (2004).

[51] O. U. Uche, S. Torquato, and F. H. Stillinger, Collective coordinate control of density distributions, Phys. Rev. E 74, 031104 (2006).

[52] S. Torquato, G. Zhang, and F. H. Stillinger, Ensemble Theory for Stealthy Hyperuniform Disordered Ground States, Phys. Rev. X 5, 021020 (2015).

[53] R. D. Batten, F. H. Stillinger, and S. Torquato, Classical disordered ground states: Super-ideal gases and stealth and equi-luminous materials, J. Appl. Phys. 104, 033504 (2008).

[54] See Supplemental Material at http://ink.aps.org/supple mental/10.1103/PhysRevApplied.15.014062 for a tabula- tion of $K-M(K)$ values, for information on the PSLL values of arrays with HUD for a varying number of elements for a range of frequencies, for a visualization of the radiation pattern of the array with HUD of 225 elements for higher operating frequencies than those shown in this manuscript, for information on the simulation and measurement setup and for the results of the analysis of arrays with HUD with required exclusion region radii.

[55] Q. Cheng, Y. Liu, H. Zhang, and Y. Hao, A generic spiral mimo array design method for short-range uwb imaging, IEEE Antennas Wirel. Propag. Lett. 19, 851 (2020).

[56] F. Liu, Y. Liu, F. Han, Y.-L. Ban, and Y. J. Guo, Synthesis of large unequally spaced planar arrays utilizing differential evolution with new encoding mechanism and cauchy mutation, IEEE Trans. Antennas Propag. 68, 4406 (2020). 